\title{
Influence of the Wavelength Dependence of Birefringence in the Generation of Supercontinuum and Dispersive Wave in Fiber Optics
}

\author{
Rodrigo Acuna Herrera \\ Facultad de Ciencias, Escuela de Fisica, Universidad Nacional de Colombia, Medellin, A.A 3840, Medellín 20036, Colombia \\ Correspondence should be addressed to Rodrigo Acuna Herrera; racuna@unal.edu.co
}

Received 19 January 2017; Accepted 23 May 2017; Published 15 June 2017

Academic Editor: Ivan D. Rukhlenko

Copyright (C) 2017 Rodrigo Acuna Herrera. This is an open access article distributed under the Creative Commons Attribution License, which permits unrestricted use, distribution, and reproduction in any medium, provided the original work is properly cited.

In this paper, we perform numerical analysis about the influence of the wavelength dependence of birefringence (WDB) in the Supercontinuum (SC) and dispersive wave (DW) generation. We study different birefringence profiles such as constant, linear, and parabolic. We see that, for a linear and parabolic profile, the generation of SC practically does not change, while this does so when the constant value of the birefringence varies. Similar situation happens with the generation of dispersive waves. In addition, we observe that the broadband of the SC increases when the Stimulated Raman Scattering (SRS) is neglected for all WDB profiles.

\section{Introduction}

The SC generation has attracted a lot of attention since it has found numerous applications in the fields of telecommunication [1], optical metrology [2], ultrafast coherence spectroscopy, and biological processes [3]. The mechanisms for SC generation in nonlinear fiber optics (FO) have been studied both numerically and experimentally [4-6]. Subsequently, SC generation techniques have brought the design of tunable ultrafast laser sources [7]. In addition, SC generation sources have been used as a simple way to generate multiwavelength optical sources [1]. It is useful for dense wavelength-division-multiplexing (WDM) telecommunications. In the generation of SC, various processes are involved such as self- and cross-phase modulation, four-wave mixing, modulation instability, soliton fission, dispersive wave generation [8], and Raman scattering [9]. All these effects can contribute to creating new frequencies within the pulse spectrum. Numerous SC generation methods have been studied to get a better understanding of the mechanisms for which it is efficiently possible to generate and develop the SC laser. Within these methods, the use of Photonic Crystal Fiber has brought a lot of interests due to its highly nonlinear optics characteristics $[10,11]$.
The SC generation can be studied with the generalized nonlinear Schrodinger equation (NLSE), which models the propagation of optical pulses in nonlinear FO. NLSE has been used to analyze the influence of various parameters on the SC generation $[12,13]$. In those studies, the WDB in the Supercontinuum generation has not been enough studied $[14,15]$. In this work, we study numerically the influence of WDB in the SC and DW generation. We analyze different birefringence profiles such as constant, linear, and parabolic. Some of those profiles can be seen in references $[16,17]$.

We show that, for a linear and parabolic profile, the generation of SC and DW do not change, while they do so as the constant value of the birefringence varies. We also found that the broadband of the SC is wider when the Stimulated Raman Scattering (SRS) is neglected for all birefringence profiles.

The paper is structured as follows. Section 2 presents the fundamental theory of nonlinear pulse propagation in birefringent fiber optics. Section 3 describes the numerical calculation of SC and DW generation for different birefringent FO profiles by using the split-step Fourier method [9] and the four-order Runge-Kutta algorithm [18]. Finally, the conclusions are presented in Section 4. 


\section{Nonlinear Pulse Propagation in Birefringent Fibers}

The nonlinear pulse propagation, in birefringent optical fiber, can be written as follows [9]:

$$
\begin{aligned}
& i \frac{\partial A_{j}}{\partial z}+\sum_{m \geq 2} \frac{i^{m-1} \beta_{m j}}{m} \frac{\partial A_{j}^{m}}{\partial T^{m}}-(-1)^{j} b A_{j} \\
& \quad=-\left(1+i \tau_{\text {shock }} \frac{\partial}{\partial T}\right)\left[\left(1-f_{R}\right) N_{j}^{1}+f_{R} N_{j}^{2}\right],
\end{aligned}
$$

where $A_{j}(j=1,2)$ corresponds to the normalized field amplitude in the $x(j=1)$ or $y(j=2)$ direction, $T=t-\beta_{1} z$ is the relative time, $t$ is the absolute time, and $z$ is the propagation length. The constants $\beta_{m j}$ are the dispersion parameters. $b=\Delta k L_{D} / 2$ is the normalized birefringence parameter, where $\Delta k=\Delta n k_{0}$ and $\Delta n$ and $k_{0}$ are the birefringence and propagation constant in vacuum, respectively. $L_{D}=T_{o}^{2} /\left|\beta_{2}\right|$ is the dispersion length, with $\beta_{2}$ being the second-order dispersion parameter and $T_{o}$ being the initial pump pulse width. $f_{R}=0.245$ is the Raman coefficient and $\tau_{\text {shock }}=1 / \omega_{o}$ is the optical shock time scale. $\omega_{o}$ is the pump frequency. The remaining terms in (1) are

$$
\begin{aligned}
N_{j}^{1}= & \left(\left|\left(A_{j}\right)\right|^{2}+\frac{2}{3}\left|\left(A_{3-j}\right)\right|^{2}\right) A_{j} \\
& +\frac{1}{3}\left(A_{j}\right)^{*}\left(A_{3-j}\right)^{2}, \\
N_{j}^{2}= & \left(A_{j}\right)\left[f_{1} \otimes\left|\left(A_{j}\right)\right|^{2}+f_{2} \otimes\left|\left(A_{3-j}\right)\right|^{2}\right] \\
& +\left(A_{3-j}\right)\left[f_{3} \otimes\left(A_{j}\left(A_{3-j}\right)^{*}+A_{3-j}\left(A_{j}\right)^{*}\right)\right],
\end{aligned}
$$

where $\otimes$ is the convolution operator, defined, for instance, as

$$
\begin{aligned}
& \left(f_{1} \otimes\left|\left(A_{j}\right)\right|^{2}\right)(z, T) \\
& \quad=\int_{-\infty}^{\infty} f_{1}\left(T^{\prime}\right)\left|A_{j}\left(z, T-T^{\prime}\right)\right|^{2} d T^{\prime} .
\end{aligned}
$$

The $f_{s}$ functions for $s=1,2,3$ in (3) are given by

$$
\begin{aligned}
& f_{1}(t)=\left(f_{a}+f_{c}\right) h_{a}(t)+f_{b} h_{b}(t), \\
& f_{2}(t)=f_{a} h_{a}(t), \\
& f_{3}(t)=\frac{\left[f_{b} h_{b}(t)+f_{c} h_{a}(t)\right]}{2} .
\end{aligned}
$$

$f_{a}=0.75, f_{b}=0.21, f_{c}=0.04$, and

$$
\begin{aligned}
& h_{a}(t)=\frac{\tau_{1}^{2}+\tau_{2}^{2}}{\tau_{1} \tau_{2}^{2}} \exp \left(\frac{-t}{\tau_{2}}\right) \sin \left(\frac{t}{\tau_{1}}\right), \\
& h_{b}(t)=\frac{2 \tau_{b}-t}{\tau_{b}^{2}} \exp \left(\frac{-t}{\tau_{b}}\right),
\end{aligned}
$$

with $\tau_{1}=12.2 \mathrm{fs}, \tau_{2}=32 \mathrm{fs}$, and $\tau_{b}=96 \mathrm{fs}$. We ignore the SRS and $\beta_{m j}$ for $m \geq 4$ in (1) in order to analyze the DW behavior. The dispersive wave generation is characterized by the parameters $\delta_{3}=\beta_{3} / 6\left|\beta_{2}\right| T_{0}$ and the soliton order $N=$ $\sqrt{L_{D} / L_{\mathrm{NL}}}$, where $\beta_{3}$ is the third-order dispersion parameter. $L_{\mathrm{NL}}=1 / P_{o} \gamma$ is the nonlinear length, where $P_{o}$ and $\gamma$ are the initial peak power of the pump and the nonlinear coefficient, respectively.

\section{Numerical Results}

For nonlinear pulse simulations, (1) is numerically solved by combining the split-step Fourier method [9] and the fourorder Runge-Kutta algorithm [18]. The basic idea is to divide the equation into a dispersive and nonlinear operator; that is,

$$
\frac{\partial A_{j}}{\partial z}=\left(\widehat{D_{j}}+\widehat{N_{j}}\right) A_{j},
$$

where

$$
\begin{aligned}
& \widehat{D_{j}}=i\left(\sum_{m \geq 2} \frac{i^{m-1} \beta_{m j}}{m} \frac{\partial A_{j}^{m}}{\partial T^{m}}-(-1)^{j} b A_{j}\right), \\
& \widehat{N}_{j}=i\left(1+i \tau_{\text {shock }} \frac{\partial}{\partial T}\right)\left[\left(1-f_{R}\right) N_{j}^{1}+f_{R} N_{j}^{2}\right] .
\end{aligned}
$$

The dispersive and nonlinear operators act together along the fiber. During numerical simulations, the fiber is divided into many $N$-sections with size $h$; at each section each operator acts independently; that is, at section $i, \widehat{N}_{j}=0$ and $\widehat{D_{j}}$ acts; at the next section $i+1, \widehat{D_{j}}=0$, and $\widehat{N_{j}}$ acts and so on. The case

$$
\frac{\partial A_{j}}{\partial z}=\widehat{D_{j}} A_{j}
$$

is solved in the Fourier domain according to

$$
A_{j}(z+h, T) \approx \exp \left(h \widehat{D_{j}}\right) A_{j}(z, T) .
$$

The exponential operator $\exp \left(h \widehat{D_{j}}\right)$ is calculated by the mathematical prescription:

$$
\begin{aligned}
& \exp \left(h \widehat{D_{j}}\right) A_{j}(z, T) \\
& \quad=F_{T}^{-1} \exp \left[h \widehat{D_{j}}(-i \omega)\right] F_{T} A_{j}(z, T),
\end{aligned}
$$

where $F_{T}$ represents the Fourier-transform operator, $\widehat{D_{j}}(-i \omega)$ is obtained from (8) by replacing $\partial / \partial T$ by $-i \omega$, and $\omega$ is the frequency in the Fourier domain. The other case

$$
\frac{\partial A_{j}}{\partial z}=\widehat{N}_{j} A_{j}
$$

is solved by using the four-order Runge-Kutta Method [18], where the following algorithm is implemented:

$$
\begin{aligned}
\frac{\partial A_{j}}{\partial z} & =f\left(z, A_{j}\right)=\widehat{N}_{j} A_{j}, \\
A_{j}^{m+1} & =A_{j}^{m}+\frac{h}{6}\left(k_{1}+2 k_{2}+2 k_{3}+k_{4}\right), \\
z_{m+1} & =z_{m}+h,
\end{aligned}
$$




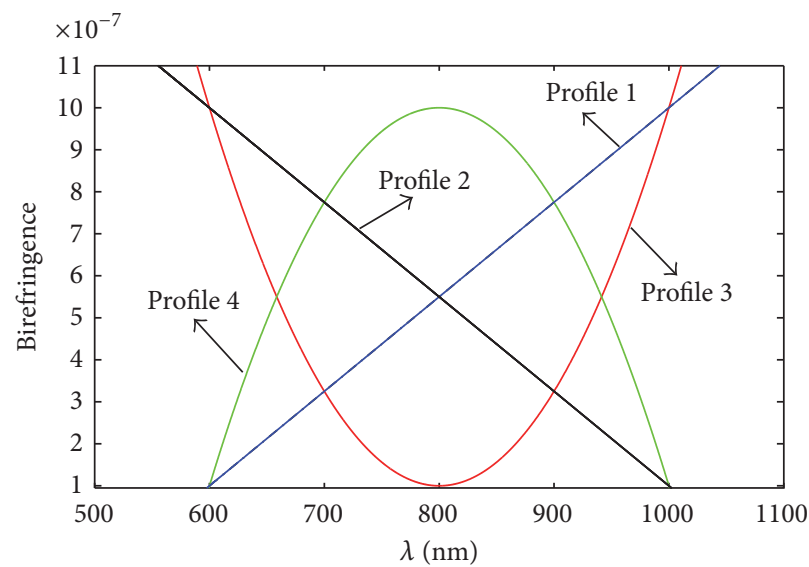

Figure 1: Birefringence profiles. Profile 1: $\Delta n=-1.25 \times 10^{-6}+2.25 \times 10^{-9} \lambda$. Profile 2: $\Delta n=2.35 \times 10^{-6}-2.25 \times 10^{-9} \lambda$. Profile $3: \Delta n=$ $10^{-7}+2.25 \times 10^{-11}(\lambda-800)^{2}$. Profile 3: $\Delta n=10^{-6}-2.25 \times 10^{-11}(\lambda-900)^{2} . \lambda$ is in nm.
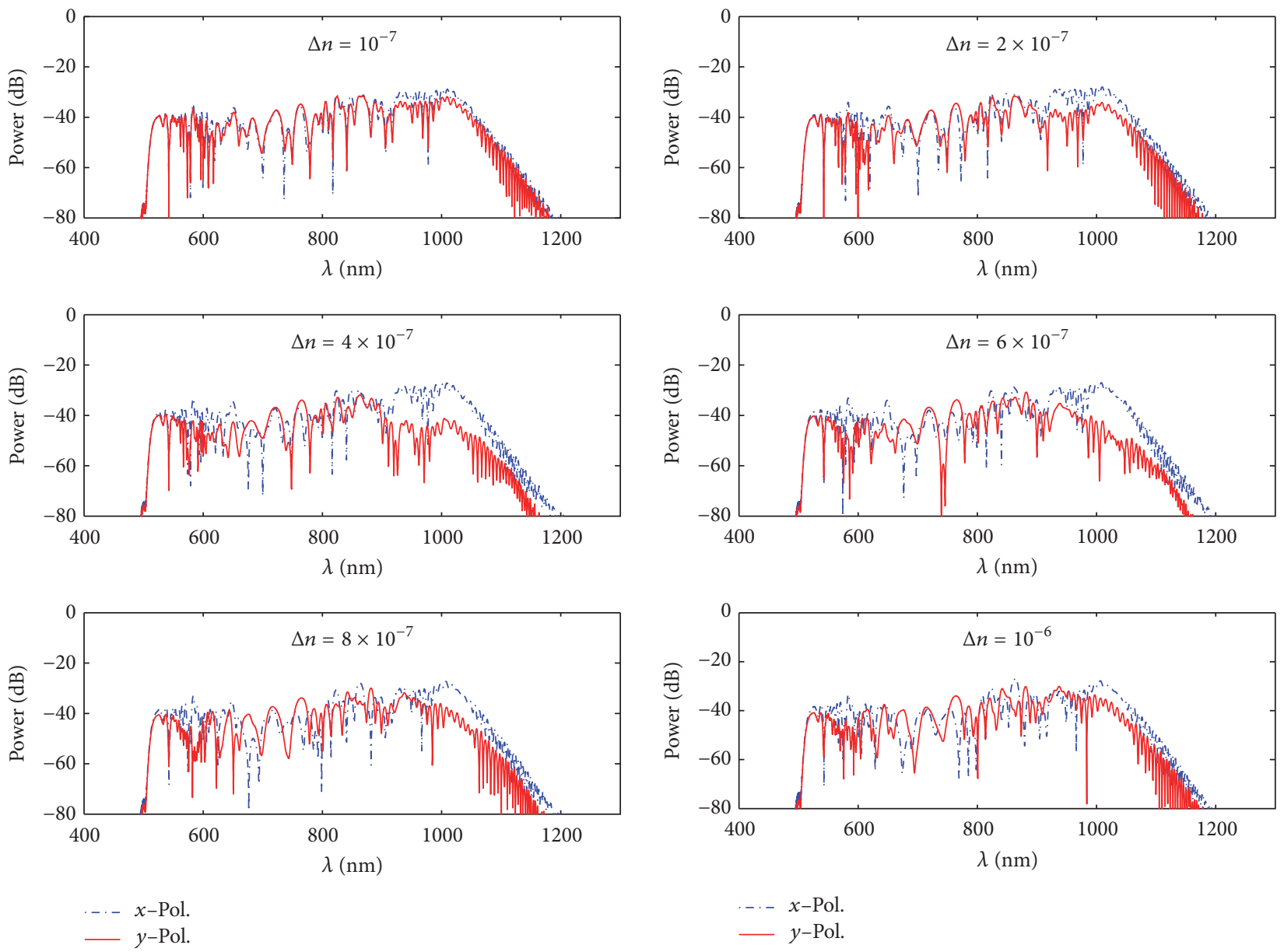

FIGURE 2: SC generation for different constant birefringence values. $\Delta n=10^{-7}, 2 \times 10^{-7}, 4 \times 10^{-7}, 6 \times 10^{-7}, 8 \times 10^{-7}$, and $10^{-6}$.

for $m=0,1,2, \ldots, N$ and $A_{j}^{0}(z=0)=A_{0} \operatorname{sech}\left(T / T_{0}\right)$, where $A_{0}=\sqrt{P_{0}}$. The constants $k_{1}, k_{2}, k_{3}$, and $k_{4}$ are given by

$$
k_{1}=f\left(z^{m}, A_{j}^{m}\right) \text {, }
$$

$$
\begin{aligned}
& k_{2}=f\left(z^{m}+\frac{h}{2}, A_{j}^{m}+\frac{h}{2} k_{1}\right), \\
& k_{3}=f\left(z^{m}+\frac{h}{2}, A_{j}^{m}+\frac{h}{2} k_{2}\right), \\
& k_{4}=f\left(z^{m}+h, A_{j}^{m}+h k_{3}\right) .
\end{aligned}
$$



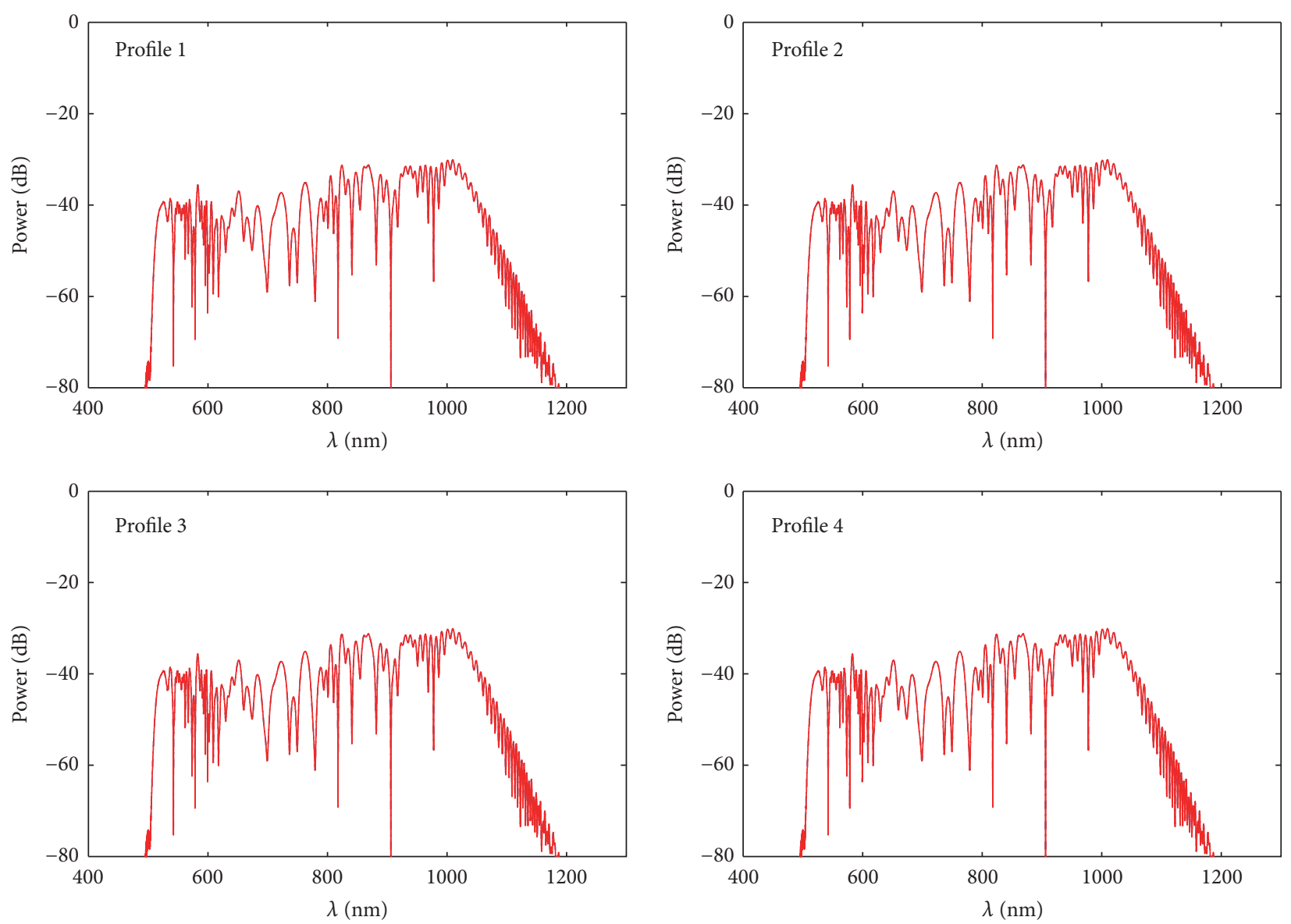

FIGURE 3: SC generation for profiles shown in Figure 1. $x$ and $y$ polarization are the same. Polarization angle $\theta=45^{\circ}$.

For our simulations, we assume the dispersion terms are the same for $x$ and $y$ polarization and use the typical parameters shown in Table 1, where the length of propagation is $6 \mathrm{~cm}$. We consider the birefringence profiles shown in Figure 1. They are linear increasing (profile 1), linear decreasing (profile 2), parabolic positive (profile 3), and parabolic negative (profile 4). We first compute the SC generation in both $x$ and $y$ polarization for six constant birefringence values between $10^{-7}$ and $10^{-6}$. The results are shown in Figure 2. We see that the generation of SC varies as the birefringence does so. For each birefringence value, we see depths at different wavelengths.

We also calculated the generation of SC for all profiles shown in Figure 1. We display the results in Figure 3. We see that all SC generations are the same and have the same two big depths around $550 \mathrm{~nm}$ and $900 \mathrm{~nm}$, which is different from constant birefringence profile (Figure 2). We repeated the above computation for $f_{R}=0$ (no SRS). The results can be viewed in Figure 4. The broadband of the SC is wider compared to the one seen in Figure 3. In addition, the biggest depth is now around $700 \mathrm{~nm}$, which tells us that SCG and depths on it are really influenced by WDB.

We finally investigated how the DW frequency can be affected by birefringence profiles. As is known, DW is a linear wave that propagates in any dispersive medium which can be
TABLE 1: Simulation parameter.

\begin{tabular}{lc}
\hline Parameter & Value \\
\hline$\gamma$ & $0.08 \mathrm{~W}^{-1} \mathrm{~m}^{-1}$ \\
$\beta_{2}$ & $-1.3504 \times 10^{-2} \mathrm{ps}^{2} \mathrm{~km}^{-1}$ \\
$\beta_{3}$ & $8.2385 \times 10^{-5} \mathrm{ps}^{3} \mathrm{~km}^{-1}$ \\
$\beta_{4}$ & $-9.1913 \times 10^{-8} \mathrm{ps}^{4} \mathrm{~km}^{-1}$ \\
$\beta_{5}$ & $1.7589 \times 10^{-10} \mathrm{ps}^{5} \mathrm{~km}^{-1}$ \\
$\beta_{6}$ & $-3.8095 \times 10^{-13} \mathrm{ps}^{6} \mathrm{~km}^{-1}$ \\
$\beta_{7}$ & $9.4138 \times 10^{-16} \mathrm{ps}^{7} \mathrm{~km}^{-1}$ \\
$P_{o}$ & $10 \mathrm{~kW}$ \\
$T_{0}$ & $50 \mathrm{fs}$ \\
$A(0, T)$ & $\sqrt{P_{o}} \mathrm{sech}\left(T / T_{o}\right)$ \\
$A_{x}(0, T)$ & $A(0, T) \cos (\theta) ; \theta$ is the polarization angle \\
$A_{y}(0, T)$ & $A(0, T) \sin (\theta)$ \\
\hline
\end{tabular}

generated from the disturbance of solitons due to the thirdorder dispersion $\beta_{3}$. The dispersive wave frequency can be obtained by a phase-matching argument requiring that the DW propagates with the same phase velocity as that of the soliton. Then, we computed the normalized DW frequency shift $\Delta v_{d} T_{0}$ and DW peak power versus $\delta_{3}$ for constant 

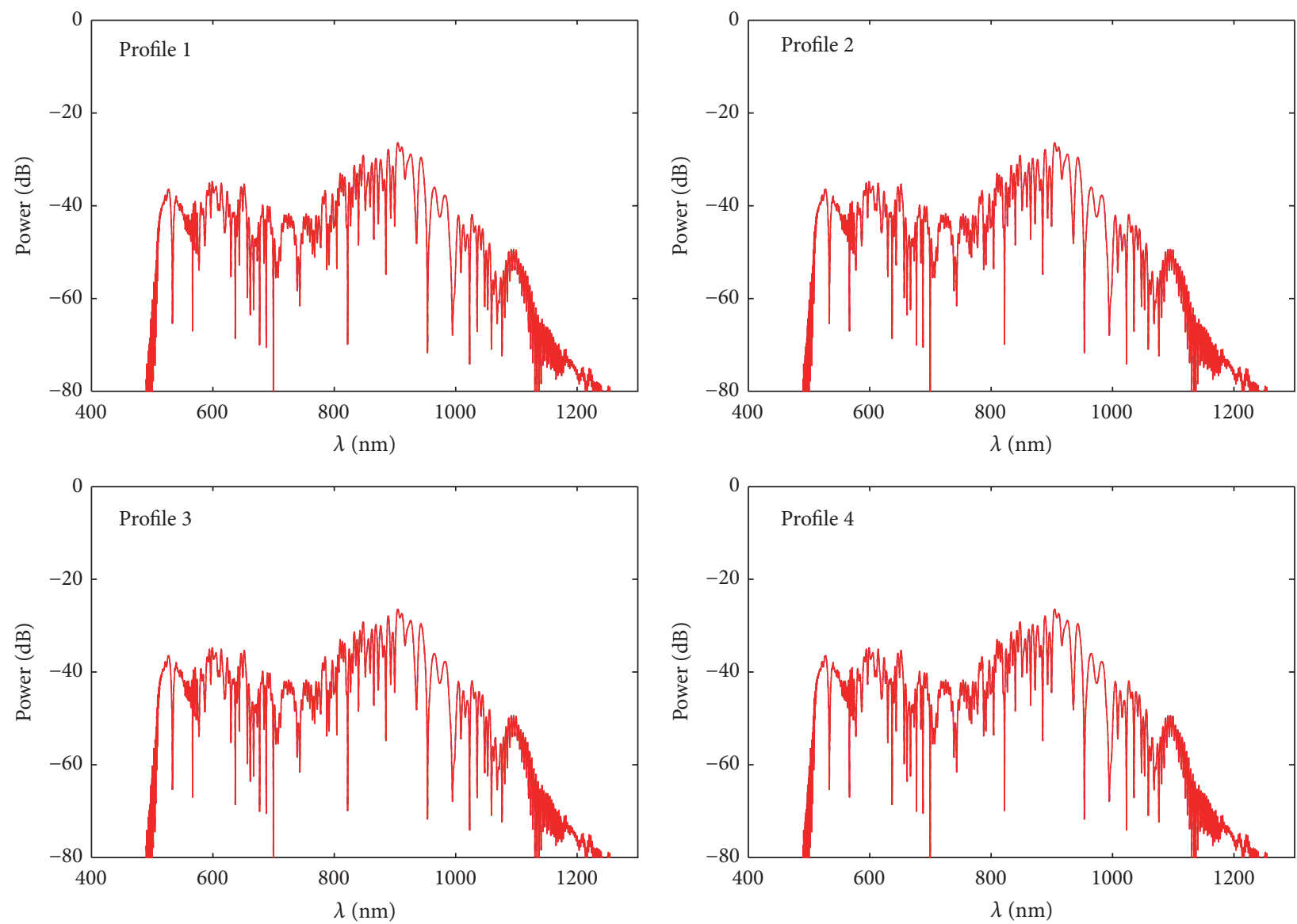

Figure 4: SC generation for profiles shown in Figure 1, with $f_{R}=0 . x$ and $y$ polarization are the same. Polarization angle $\theta=45^{\circ}$.

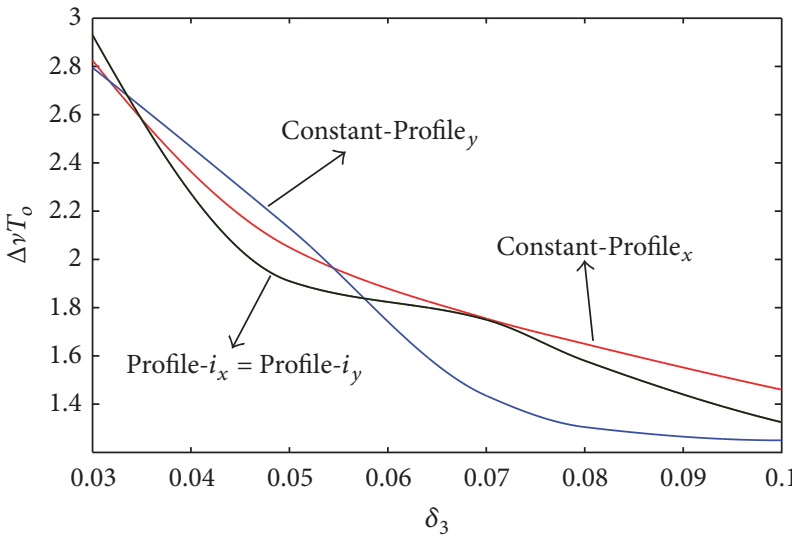

FIGURE 5: DW generation for constant birefringence profile and the ones shown in Figure 1. Profile- $i_{x}=$ Profile- $i_{y}$, with $i=1,2,3,4$. Polarization angle $\theta=45^{\circ}$.

birefringence profile and the ones shown in Figure 1. We set $N=2$, which corresponds to the second-order soliton. DW frequency shift is defined as $\Delta v_{d}=\left(v_{d}-v_{s}\right)$, where $v_{d}$ is the DW frequency and $v_{s}$ is the soliton frequency. We performed simulations and had the results shown in Figures 5 and 6 . The DW characteristics are the same for any WDB profile, but different from constant profile. In addition,

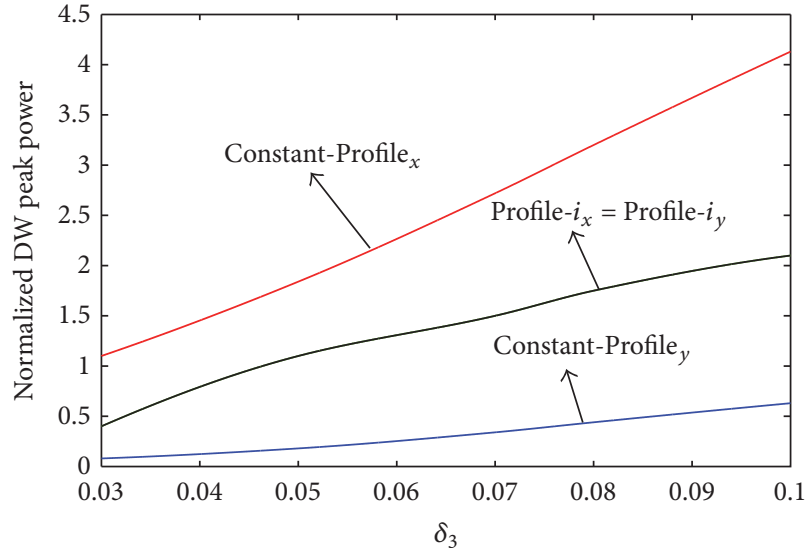

FIGURE 6: DW peak power for constant birefringence profile and the ones shown in Figure 1. Profile- $i_{x}=$ Profile- $i_{y}$, with $i=1,2,3,4$. Polarization angle $\theta=45^{\circ}$.

the $x$ - and $y$-DW polarizations have equal behavior for all WDB. From Figure 6, the normalized DW peak power almost scales linearly to $\delta_{3}$, which means that this property does not depend on WDB. The above effects can be used to control the generation of the dispersive wave created from solitons using the WDB. 


\section{Conclusion}

We have shown that the generation of SC and DW properties do not depend on the four WDB profiles seen in Figure 1. We observed that there are a couple of big depths around $500 \mathrm{~nm}$ and $900 \mathrm{~nm}$, while they are at different positions for constant birefringence profile depending on its value. The generation of DW has the same behavior for any WDB profile, but different from the constant ones. Finally, we have proven that the broadband of SC is bigger for WDB when SRS is neglected.

\section{Conflicts of Interest}

The author declares that there are no conflicts of interest regarding the publication of this paper.

\section{Acknowledgments}

The author acknowledges support from the Universidad Nacional de Colombia (Grant no. 35453) and COLCIENCIAS (Grant no. FP44842-107-2016) for this work.

\section{References}

[1] H. Takara, T. Ohara, K. Mori et al., "More than 1000 channel optical frequency chain generation from single supercontinuum source with $12.5 \mathrm{GHz}$ channel spacing," Electronics Letters, vol. 36, no. 25, pp. 2089-2090, 2000.

[2] D. J. Jones, S. A. Diddams, J. K. Ranka et al., "Carrier-envelope phase control of femtosecond mode-locked lasers and direct optical frequency synthesis," Science, vol. 288, no. 5466, pp. 635639, 2000.

[3] "Topics in Applied Physics," in Ultrafast Laser Pulses and Applications, W. Kaiser, Ed., vol. 60, Springer, Heidelberg, Berlin, Germany, 1988.

[4] A. V. Husakou and J. Herrmann, "Supercontinuum generation of higher-order solitons by fission in photonic crystal fibers," Physical Review Letters, vol. 87, no. 20, Article ID 203901, 2001.

[5] S. Coen, A. H. L. Chau, R. Leonhardt et al., "White-light supercontinuum generation with 60-ps pump pulses in a photonic crystal fiber," Optics Letters, vol. 26, no. 17, pp. 1356-1358, 2001.

[6] J. M. Dudley, G. Genty, and S. Coen, "Supercontinuum generation in photonic crystal fiber," Reviews of Modern Physics, vol. 78, no. 4, pp. 1135-1184, 2006.

[7] M. Nisoli, S. De Silvestri, and O. Svelto, "Generation of high energy 10 fs pulses by a new pulse compression technique," Applied Physics Letters, vol. 68, no. 20, pp. 2793-2795, 1996.

[8] Z. Zhu and T. G. Brown, "Polarization properties of supercontinuum spectra generated in birefringent photonic crystal fibers," Journal of the Optical Society of America B, vol. 21, no. 2, p. 249, 2004.

[9] G. P. Agrawal, Nonlinear optics, Academic Press, 4th edition, 2007.

[10] J. K. Ranka, R. S. Windeler, and A. J. Stentz, "Visible continuum generation in air-silica microstructure optical fibers with anomalous dispersion at $800 \mathrm{~nm}$," Optics Letters, vol. 25, no. 1, pp. 25-27, 2000.
[11] J. C. Knight, T. A. Birks, P. St. J. Russell, and D. M. Atkin, "Allsilica single-mode optical fiber with photonic crystal cladding," Optics Letters, vol. 21, no. 19, pp. 1547-1549, 1996.

[12] J. M. Dudley and S. Coen, "Numerical simulations and coherence properties of supercontinuum generation in photonic crystal and tapered optical fibers," IEEE Journal on Selected Topics in Quantum Electronics, vol. 8, no. 3, pp. 651-659, 2002.

[13] J. M. Dudley and S. Coen, "Coherence properties of supercontinuum spectra generated in photonic crystal and tapered optical fibers," Optics Letters, vol. 27, no. 13, pp. 1180-1182, 2002.

[14] S. Roy, S. K. Bhadra, and G. P. Agrawal, "Dispersive waves emitted by solitons perturbed by third-order dispersion inside optical fibers," Physical Review A - Atomic, Molecular, and Optical Physics, vol. 79, no. 2, Article ID 023824, 2009.

[15] R. A. Herrera, "Nonlinear pulse propagation in wavelength dependence of birefringence fiber optics," Applied Optics, vol. 54, no. 13, pp. 3933-3936, 2015.

[16] L. Labonté, D. Pagnoux, P. Roy, F. Bahloul, and M. Zghal, "Numerical and experimental analysis of the birefringence of large air fraction slightly unsymmetrical holey fibres," Optics Communications, vol. 262, no. 2, pp. 180-187, 2006.

[17] A. Michie, J. Canning, K. Lyytikäinen, M. Åslund, and J. Digweed, "Temperature independent highly birefringent photonic crystal fibre," Optics Express, vol. 12, no. 21, pp. 5160-5165, 2004.

[18] K. E. Atkinson, An Introduction to Numerical Analysis, John Wiley \& Sons, New York, NY, USA, 2nd edition, 1989. 




Advances in

Operations Research

vatersals

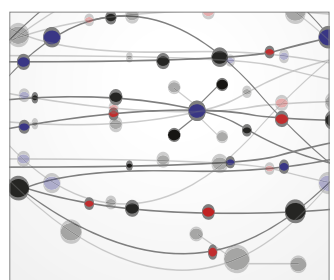

\section{The Scientific} World Journal

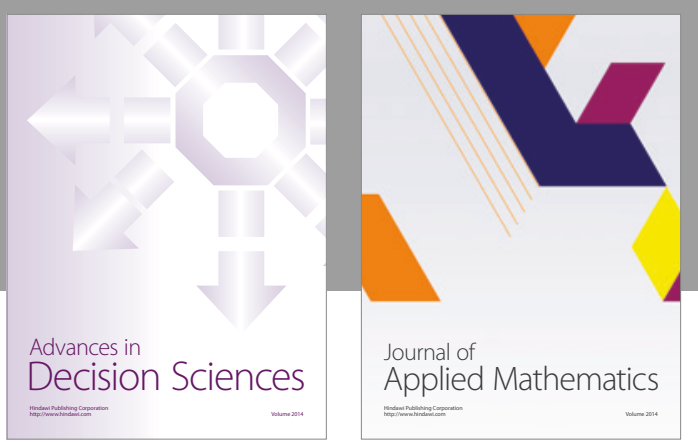

Algebra

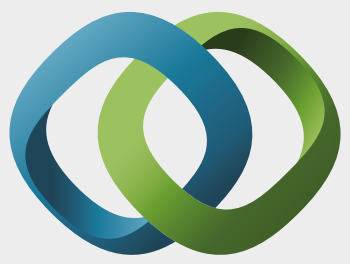

\section{Hindawi}

Submit your manuscripts at

https://www.hindawi.com
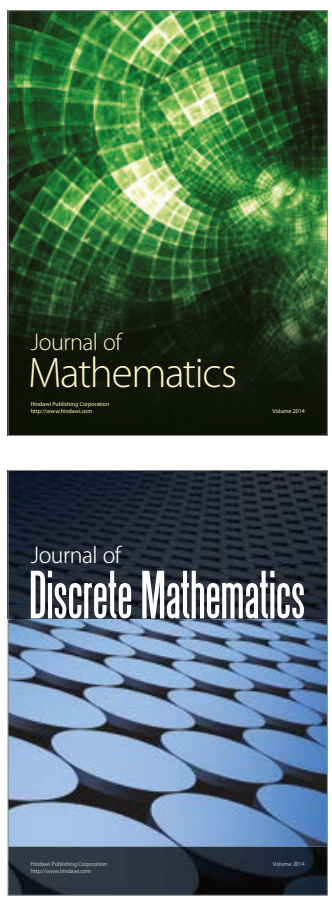

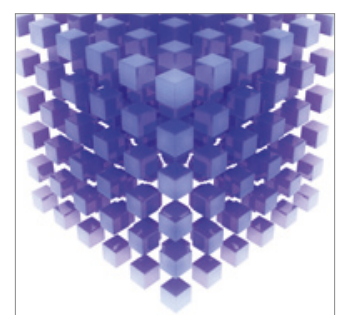

Mathematical Problems in Engineering
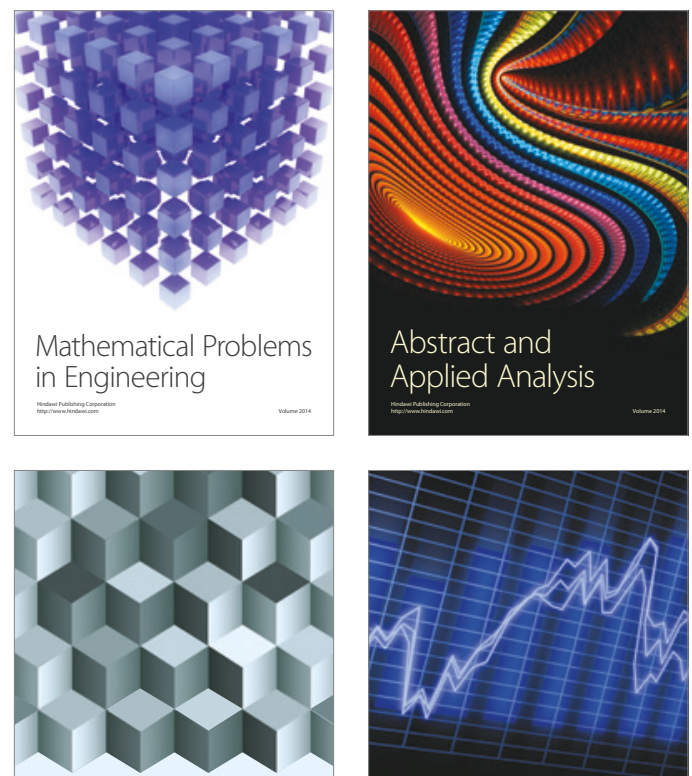

Journal of

Function Spaces

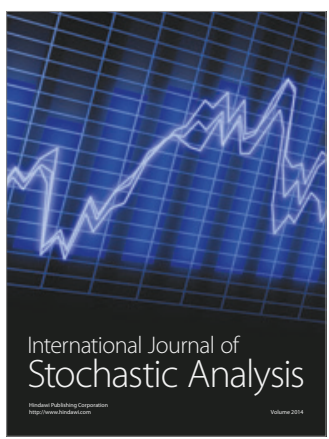

Probability and Statistics
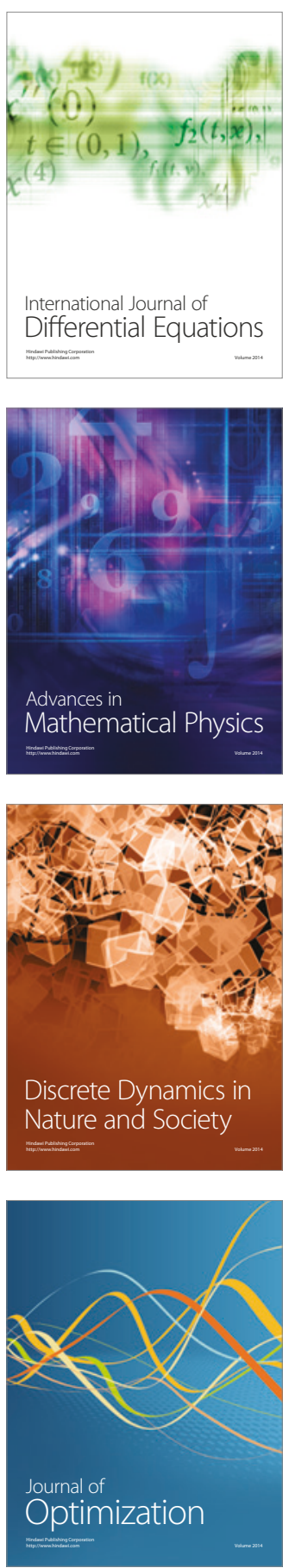